\title{
Eye Gaze Interaction for Supporting Creative Work with Disabled Artists
}

\author{
Dr Chris Creed \\ Digital Humanities Hub \\ University of Birmingham, B15 2TT \\ creedcpl@bham.ac.uk
}

\begin{abstract}
Key findings are presented around work-in-progress with ten disabled artists examining the use of eye gaze tracking "out-of-the-box" to control artistic software. Future work will explore how new interface paradigms can better support artists using assistive technology for creative work.
\end{abstract}

Assistive Technology. Digital Accessibility. Disabled Artists. Eye Gaze Tracking.

\section{INTRODUCTION}

Disabled artists with physical impairments can experience significant issues when using artistic tools such as brushes, pencils, and other materials. Traditional assistive tools (e.g. head wands and mouth sticks) can help facilitate artistic work, but also introduce further issues such as chronic neck strain and damage to teeth. Moreover, research has shown that people with severe physical impairments need significant support from others when doing creative work around setting up tools, moving materials, and being available to make any further adjustments (Perera et al., 2009). This lack of independence can be particularly frustrating for disabled artists and can stifle creative flow.

Digital technologies have the potential to address many of the barriers disabled artists experience, yet little work has focused on this important research area. To address the lack of work, we are exploring the current practice of disabled artists and the new opportunities novel digital technologies (e.g. eye gaze tracking, mid-air gesturing, voice recognition, etc.) offer around supporting artistic practice. This collaborative research is being undertaken by the Digital Humanities Hub (University of Birmingham), DASH (a disability arts organisation), the University of Chicago at Illinois, Arts Council England, special needs colleges (including the charity SCOPE's Beaumont College and National Star College), and the School of Art at Birmingham City University.

This multi-disciplinary approach has helped to inform our research focus and the types of technologies we are investigating. For instance, eye gaze tracking technology offers a novel method for producing creative work and its recent drop in price (i.e. budget eye trackers are now available for under £100) makes it much more accessible to a disabled audience. However, the majority of research around eye gaze technology over the past 20 years has primarily focused around implementation details of the technology (e.g. algorithms for optimising the tracking of eye movements), its use in areas such as eye typing (Majaranta and Räihä, 2007), or as a tool for measuring the usability of interfaces (Jacob and Kam, 2003). There has been no work, however, that has investigated the potential of the technology to support the creative process of professional disabled artists.

This paper therefore provides an overview of workin-progress that is exploring the potential of eye tracking technology to support creative work. In particular, we focus on initial research that involved working with ten disabled artists to examine whether the technology "out-of-the-box" can be used with standard digital art software (i.e. Photoshop). We highlight the key findings from this research and limitations that need to be addressed.

\section{STUDY DESIGN}

The study was performed in a lab-based environment at the University of Birmingham where artists were seated in front of a monitor that had an eye tracker (the Tobii EyeX) attached to it in addition to a laptop with two switches (i.e. large buttons). The first switch emulated a left mouse click and the other enabled the user to pick up and drop objects (i.e. move objects around).

Artists were asked to complete four basic tasks with Photoshop using their eyes - (T1) move a square from one side of the screen to the other, 
(T2) select four icons from the Photoshop tool menu, (T3) create a new square to a specified size, and (T4) perform multiple manipulations on a single shape (i.e. resize, rotate, and drag). Artists and their personal assistants were encouraged to share their thoughts via a "think-aloud" protocol. We also conducted semi-structured interviews with artists to explore their perceptions of the technology. All interactions were videoed for later analysis.

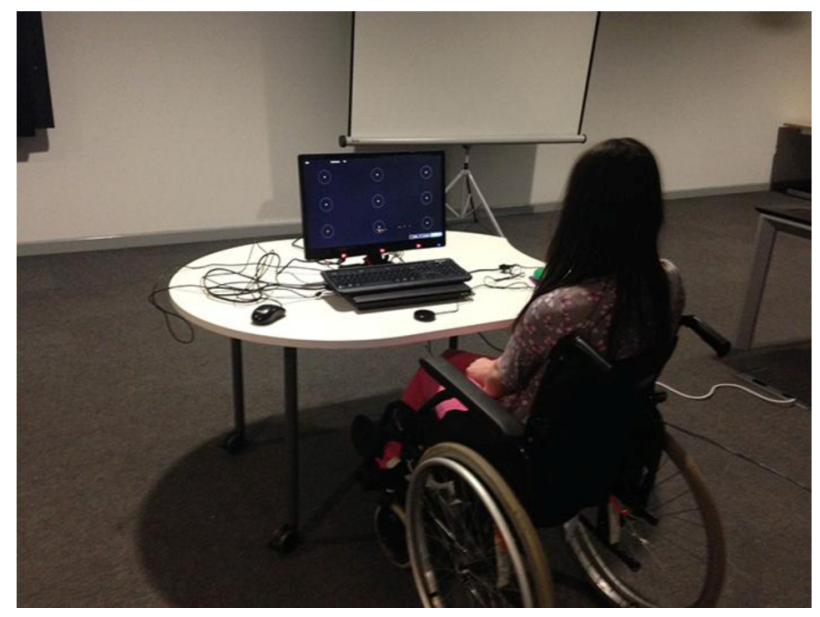

Figure 1: An artist completing the eye calibration process (following a dot around the screen)

\section{RESULTS}

Ten disabled artists (9 female) participated in the study and included painters, illustrators, printmakers, and digital photographers. Artists varied in career stage from emerging to mid-career and more established artists. All of the artists had physical impairments related to conditions such as multiple sclerosis, motor neurone disease, generalised dystonia, muscular dystrophy, cerebral palsy, arthrogryposis, quadriplegia, and multiple joint arthritis. Several key themes emerged from this work:

Frustrating User Experience: It took artists a significant amount of time to attempt each of the tasks. For instance, it took $75 \%$ of the artists over 15 seconds to complete T1 with times ranging from 12 seconds to $3: 39$ (none were able to place the square in exactly the correct position). A similar theme was observed for the other tasks - in particular, none of the artists were able to successfully complete T4. As such, the length of time required to complete simple tasks was clearly a source of frustration for artists.

Cursor Sensitivity: Several artists commented that one of the main interaction issues was the cursor was too sensitive. The high level of sensitivity made it difficult for participants to have sufficient control when performing actions that required finer levels of movement. For instance, when attempting to create a new square with specific dimensions in
T3 (via a dragging movement), the outline of the new square would jump around in relation to the user's eye movement.

Selection of Small Icons: Many of the artists had issues in selecting small icons within the Photoshop interface. In T3, for example, artists experienced significant difficulties in placing the cursor over icons such as the select, rectangle, and eraser tools. Another significant issue (in T4) was selecting the small manipulation handles that are placed around an object that enable it to be resized or rotated. These are easy to manipulate with a mouse (if you do not have a physical impairment), but clearly much more problematic when using our eyes. This ultimately meant that none of the artists were able to complete $\mathrm{T} 4$.

Switches: Three of the artists with more severe motor impairments had issues in using the switches primarily because they needed to look at them and make a focused effort to press them. This will clearly cause issues when using eye gaze technology as users will be directing their gaze away from the screen. Some artists placed the switch on their lap which helped to make selections easier. Dwell based interfaces where selections are made via looking at an object for a set period of time can help to overcome this issue.

Eye Gaze for Wider Practice: Several of the artists felt this technology would be more suitable in supporting wider tasks around artistic practice such as email correspondence, ordering supplies, managing budgets, researching online, etc. (i.e. the "business" side of their practice). This is a crucial point as these types of tasks typically require a mouse and keyboard which they struggle to use effectively. Time spent on these tasks ultimately results in less time for creative activities.

Physical Issues: Several of the artists commented that they experienced tiredness, eye strain, and tension in their heads after completing the tasks. This can be a common experience after initial use, but tends to diminish over time. Two artists had a tendency to move closer to the screen and sensor when completing the tasks - this has the potential to cause lower back strain and discomfort. It is therefore essential that the calibration process is completed whilst the user is in a comfortable and relaxed position.

\section{CONCLUSION}

This initial work has highlighted the limitations around using eye tracking "out-of-the-box" with standard artistic software. It is clear that "bolting" this technology onto existing platforms (typically designed for mouse and keyboard interactions) is unlikely to be an optimal approach. We are now collaboratively investigating with partners and 
disabled artists how new creative interaction designs can better support people using eye gaze tracking and other technologies for creative work.

\section{REFERENCES}

Jacob, R. J. \& Karn, K. S. (2003) Eye tracking in human-computer interaction and usability research: Ready to deliver the promises. Mind, 2(3), 4.

Majaranta, P. \& Räihä, K. J. (2007) Text entry by gaze: Utilizing eye-tracking. In Text entry systems: Mobility, accessibility, universality. 175-187.

Perera, D., Eales, R. J., and Blashki, K. (2009) Supporting the creative drive: investigating paralinguistic voice as a mode of interaction for artists with upper limb disabilities. Universal Access in the Information Society, 8,(2). 77-88. 